BULL. AUSTRAL. MATH. SOC.

$60 F 05,60 G 42$

VOL. $22(1980), 469-471$.

\title{
Martingale RATES AND \\ WEAKLY EXCHANGEABLE ARRAYS
}

NeVille C. Weber

This dissertation can be divided into two sections. The first, Chapters 1 and 2, is concerned with weak limit theorems and rates of convergence for forward and reversed martingales while in Chapters 3 to 5 some of the results of the earlier chapters are used to obtain limit theorems for various weakly exchangeable arrays.

Specifically, Chapter 1 is concerned with central limit theorems for martingales. $L_{\infty}$ and $L_{p}$ bounds are provided for the departure from normality of martingales. These results provide a generalisation of the results obtained by Heyde and Brown [3] and Ibragimov [4]. The chapter is concluded by using the martingale representation of stationary processes to obtain rates of convergence for a general stationary ergodic sequence of random variables.

The first part of Chapter 2 provides a series of distributional limit results for reversed martingales. The reversed martingale analogues of martingale central limit theorems and invariance principles are given for their own interest and to provide the necessary limit results that are required in later chapters. In the second part of Chapter 2 reversed martingale rate results analogous to those given for forward martingales, are developed.

In Chapter 3 the concept of a weakly exchangeable array is introduced and reversed martingale methods are used to prove a strong law of large

Received 25 August 1980. Thesis submitted to the University of Sydney, August 1979. Degree approved August 1980. Supervisor: Dr M.P. Quine. 
numbers, central limit theorem and invariance principle for a sum of random variables taken from such an array. These theorems generalise the corresponding results for $U$-statistics and they generalise the work of Silverman [5] on exchangeably dissociated arrays. $L_{\infty}$ and $L_{p}$ rates of convergence of $O\left(n^{-\frac{1}{2}} \log n\right)$ are given for the central limit theorem for a sum based on a weakly exchangeable array.

Chapter 4 analyses the central limit theorem for sequences of weakly exchangeable arrays. In the first section of this chapter a martingale approach is used to develop central limit theorems for an array of random variables, each row of which is an exchangeable set of random variables. The results given for arrays with non-random row sums generalise those of Chernoff and Teicher [2]. The central limit theorem for arrays with rows which are infinitely exchangeable sets are obtained without utilising the conditional independence structure of such sets. The martingale methods developed for these arrays are adapted in the latter part of Chapter 4 to obtain various central limit theorems for sequences of weakly exchangeable arrays of order 2 .

The final chapter of this thesis is concerned with those sequences of weakly exchangeable arrays which are generated by $U$-statistics with varying kernels. A central limit theorem and invariance principle are given for these statistics using the projection technique. A representation for weakly exchangeable arrays of order 2 , developed by Aldous [1], is used to obtain the Berry-Esseen result and $L_{p}$ rates for certain sequences of weakly exchangeable arrays of order 2 , including $U$-statistics with varying kernels of degree 2 . An Edgeworth expansion is also given for certain sequences of weakly exchangeable arrays. The thesis is concluded by giving two examples of U-statistics with varying kernels which appear in the analysis of spatial data. One statistic based on interpoint distances is used as the basis for a test of clustering and the other statistic based on the largest angle in each triad of points has been used as a basis for a test of collinearity. 


\section{References}

[1] D.J. Aldous, "Representations for partially exchangeable arrays of random variables", submitted.

[2] H. Chernoff and H. Teicher, "A central limit theorem for sums of interchangeable random variables", Ann. Math. Statist. 29 (1958), $118-130$.

[3] C.C. Heyde and B.M. Brown, "On the departure from normality of a certain class of martingales", Ann. Math. Statist. 41 (1970), 2161-2165.

[4] I.A. Ibragimov, "A central limit theorem for a class of dependent random variables", Theory Probab. App2. 8 (1963), 83-89.

[5] B.W. Silverman, "Limit theorems for dissociated random variables", Adv. in App 2. Probab. 8 (1976), 806-819. 